\title{
HIPERTENSÃO ARTERIAL: ADESÃO AO TRATAMENTO
}

\author{
ARTERIAL HYPERTENSION: TREATMENT ADHERENCE
}

Antônio Carlos de Souza Spinelli1,2

\section{RESUMO}

A hipertensão arterial, patologia que atinge $32,5 \%$ dos brasileiros adultos e mais que $60 \%$ da população de idosos, tem contribuição direta ou indiretamente em $50 \%$ das mortes por doenças cardiovasculares. Na atualidade existe a disponibilidade de vários medicamentos anti-hipertensivos que são bem tolerados, apresentando comprovado nível de potência e motivo de estudos e meta-análises que mostram importante ação na redução do risco de eventos cardiovasculares. Não obstante esses conhecimentos apenas 59\% recebem tratamento regular e só aproximadamente $31 \%$ a $34 \%$ desses se encontram com a pressão arterial nas metas de controle recomendadas nas diretrizes. A falta de controle pressórico na maioria dos casos está correlacionada a uma reduzida adesão ao tratamento farmacológico e menor ainda ao não farmacológico. A adesão ao tratamento definida como o grau de cumprimento das medidas terapêuticas indicadas para manter o controle da pressão arterial, deve sempre contemplar a vontade participativa do cada indivíduo. Os fatores implicados na adesão ao tratamento apresentam inúmeras variáveis associadas à característica da doença, relação médico-paciente, esquemas terapêuticos complexos, efeitos indesejáveis dos medicamentos e aspectos socioeconômicos, assim, diversas estratégias podem resultar em aumento da adesão ao tratamento. Além das medidas tradicionais, podem elevar a adesão uma postura mais humana e atenciosa por parte do médico, a identificação das características da população assistida, a individualização das recomendações a cada paciente e, se possível, adotar um acompanhamento com uma equipe multidisciplinar.

Descritores: Hipertensão Arterial; Adesão ao tratamento; Estratégias para a Adesão.

\begin{abstract}
Arterial hypertension, a condition that affects $32.5 \%$ of adult Brazilians and more than $60 \%$ of the elderly population, directly or indirectly contributing to 50\% of deaths from cardiovascular diseases. Currently, there are several anti-hypertensive drugs available that are well tolerated, with a proven level of potency and reason for studies and meta-analyzes that show an important action in reducing the risk of cardiovascular events. Despite this knowledge, only $59 \%$ receive regular treatment and only approximately $31 \%$ to $34 \%$ of them have blood pressure in the control goals recommended in the guidelines. The lack of pressure control in most cases is correlated with reduced adherence to pharmacological treatment and even less to non-pharmacological treatment. Adherence to treatment, defined as the degree of compliance with the therapeutic measures indicated to maintain blood pressure control, must always contemplate the participatory will of each individual. The factors involved in adherence to treatment have numerous variables associated with the characteristic of the disease, doctor-patient relationship, complex therapeutic regimens, undesirable effects of medications and socioeconomic aspects, thus, several strategies can result to increase adherence to treatment. In addition to traditional measures, a more humane and caring attitude from the doctor can increase adherence, identification of the characteristics of the assisted population, individualization of recommendations to each patient and, if possible, adopting a follow-up with a multidisciplinary team can increase compliance.
\end{abstract}

Keywords: Hypertension; Treatment Adherence;Strategies.

\section{CONSIDERAÇÕES INICIAIS}

A hipertensão arterial (HAS) é uma patologia que atinge $32,5 \%$ dos brasileiros adultos, com uma prevalência maior que $60 \%$ da população de idosos, contribuindo direta ou indiretamente para $50 \%$ das mortes por doenças cardiovasculares. ${ }^{1}$ Representa uma patologia que afeta cerca de 1,2 bilhão de pessoas no mundo. ${ }^{2}$ e aproximadamente 40 milhões no Brasil. ${ }^{1}$

O objetivo primordial do tratamento da hipertensão é reduzir a incidência de eventos cardiovasculares; para esse fim dispomos na atualidade fármacos anti-hipertensivos que além de bem tolerados, apresentam potencia comprovada isoladamente ou em combinações. Estudos e meta-análises

1. Universidade Federal do Rio Grande do Norte, Natal, RN, Brasil.

2. Cardiocentro, Natal, RN, Brasil.

Correspondência: Antônio Carlos de Souza Spinelli. Rua Campos Sales, 742 Tirol. CEP 5902O-300. Natal, RN, Brasil. spinelli@cardiol.br

http://dx.doi.org/10.47870/1519-7522/2020270118-22 
apontam que o uso desses fármacos leva a uma redução do risco de eventos cardiovasculares resultando em atenuação na morbidade e mortalidade cardiovascular, ${ }^{3}$ não obstante o que se observa na população de pacientes hipertensos é que apenas 59\% recebe tratamento regular e só aproximadamente $31 \%$ a $34 \%$ desses, apresentam pressão arterial nas metas de controle recomendadas nas diretrizes. . $^{4,5}$

A falta de controle pressórico na maioria dos casos está correlacionada a uma reduzida adesão ao tratamento, e ações mais efetivas sobre os fatores causais da não adesão são fundamentais para prevenir a mortalidade cardiovascular e uma série de eventos adversos, como hospitalizações, procedimentos de revascularização, ${ }^{6}$ hemodiálise, e o alto custo associado. ${ }^{7}$

Quando nos debruçamos sobre o tratamento não farmacológico encontramos taxas de adesão ainda mais decepcionantes, descortinando uma área importante com possibilidade de ganhos na prevenção das doenças cardiovasculares. Para isso medidas vigorosas precisam ser adotadas pelos profissionais no atendimento aos pacientes, visando elevar a adesão.

\section{CONCEITO DE ADESÃO}

Publicações especializadas no estudo dos fatores determinantes da adesão estabeleceram um conceito de adesão ao tratamento como o grau de cumprimento das medidas terapêuticas indicadas, sejam elas medicamentosas ou não, com o objetivo de manter a pressão arterial nas metas de controle estabelecidas pelas diretrizes, ressaltando-se que devem sempre reconhecer e contemplar a vontade do indivíduo em participar e colaborar com seu tratamento. ${ }^{8}$ É uma situação onde o comportamento do paciente coincide com a recomendação médica ou aconselhamento de um profissional da saúde, seguindo a prescrição medicamentosa, orientações sobre dieta, mudanças de hábitos de vida e comparecimento às consultas agendadas.

\section{FATORES IMPLICADOS NA ADESÃO AO TRATAMENTO}

Não existe um teste padrão para aferir a adesão ao tratamento, na prática realizamos a observação do comportamento dos pacientes objetivando estimar nível de colaboração participativa. São aceitos como indicadores de adesão:

1. Condição clínica do avaliado: Níveis tensionais controlados, redução na frequência de internações ou dos atendimentos em pronto-socorro.

2. Diretos: Análise de exames complementares, entre eles os que proporcionam medidas da pressão arterial fora do ambiente de consultório (MAPA e MRPA) e exames que identificam dano a órgãos alvo (determinação da albuminúria, ecocardiograma e a avaliação dos parâmetros centrais).

3. Indiretos: Contagem de comprimidos, número de receitas dispensadas.

4. Subjetivos: Relatos do paciente, familiares ou cuidadores.

5. Utilização dos serviços de saúde: Comparecimentos às consultas e avaliações aprazadas.

Os estudos dos fatores implicados na adesão ao tratamento da hipertensão arterial apontam a influência de inúmeras variáveis que estão ligadas a: característica da evolução da doença, relação médico-paciente, esquemas terapêuticos complexos, efeitos indesejáveis dos medicamentos e aspectos socioeconômicos. ${ }^{9}$ Um estudo realizado no Brasil demonstrou que pacientes que permaneciam comparecendo às avaliações de controle, após um período de 12 meses de evolução, tinham uma taxa de abandono da terapêutica decrescente e quanto maior o tempo de seguimento dos pacientes, o incremento na taxa de abandono do tratamento era cada vez menor. ${ }^{10}$ A manutenção de um seguimento de longo prazo, com uma rotina periódica de consultas e revisões clínicas, se apresenta como um fator positivo para a adesão às recomendações médicas.

A observação de forma recorrente dos fatores acima relatados culminou numa publicação da Organização Mundial da Saúde (OMS), caracterizando a adesão como um fenômeno multidimensional onde encontramos a interação de cinco fatores, que foram denominados de "dimensões", ficando evidente que os fatores relacionados ao paciente representam apenas um destes determinantes (Figura 1); assim cai por terra o equivocado conceito que os pacientes são os únicos responsáveis pelo seu tratamento. ${ }^{11}$

\section{FATORES DE ADESÃO RELACIONADOS À DOENÇA}

Um percentual significante dos pacientes hipertensos, assim como ocorre em todos portadores de doenças de evolução crônica, não apresenta uma adequada percepção de doença em relação ao seu estado de saúde. ${ }^{12}$ A hipertensão arterial é uma patologia que predominantemente cursa assintomática e muitas vezes o aparecimento de sintomas ocorre após o inicio de utilização da medicação anti-hipertensiva. ${ }^{13}$ Os pacientes que conseguem perceber na elevação da pressão arterial um fator determinante para o aumento do risco de desenvolver doenças cardiovasculares, adotam um perfil mais aderente ao tratamento. A má adesão ocorre entre aqueles que não alcançam essa compreensão.

\section{FATORES DE ADESÃO RELACIONADOS AO MÉDICO}

Questionários aplicados para aferir a adesão ao tratamento medicamentoso da hipertensão arterial revelaram que um número elevado de respondedores costuma ter uma menor

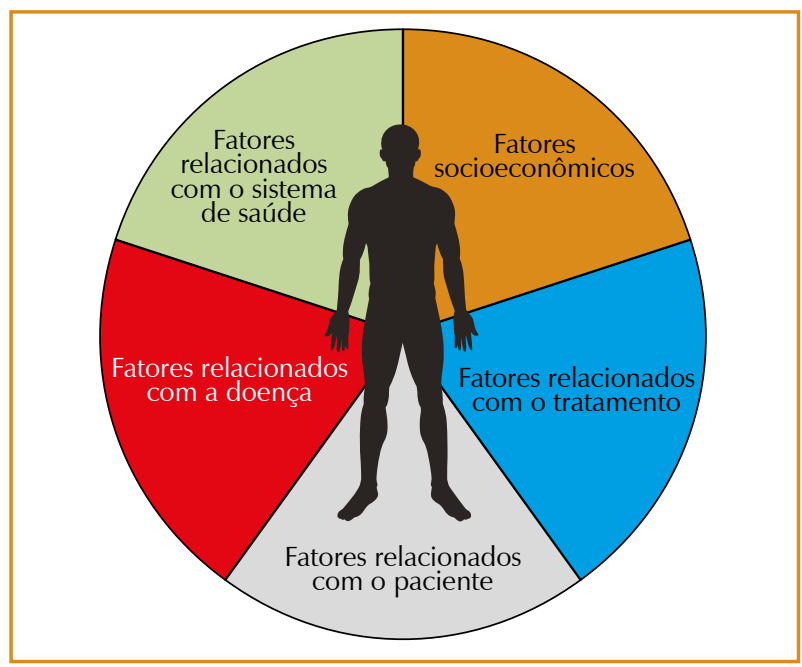

Figura 1. As cinco dimensões da adesão (OMS). 
atenção às recomendações proferidas por médicos jovens, generalistas e do sexo masculino. A análise destes questionários também permitiu identificar os principais fatores que frequentemente levam a uma inadequada sintonia medico/ paciente resultando num obstáculo à adesão. ${ }^{14}$ São situações muito corriqueiras na prática clínica, e as citações mais frequentes foram:

- Elevado tempo de espera para o atendimento.

- Relação médico-paciente precária nas consultas.

- Linguagem inadequada para o entendimento da doença e orientações.

- Excesso de informações em um tempo curto de consulta.

- Utilização de orientação verbal e muito rápida.

Imprescindível se faz para uma melhor adesão às recomendações médicas a existência de uma relação saudável e com evidente empatia durante as consultas; não basta ao profissional ser tecnicamente competente, ele precisa ter um pouco de humanidade para oferecer apoio e dedicação aos seus pacientes.

\section{FATORES DE ADESÃO RELACIONADOS AO PACIENTE}

As observações do acompanhamento de pacientes hipertensos ao longo do tempo permitiram traçar um perfil para aqueles que mostravam maior aderência às recomendações. ${ }^{15}$ Os itens mais frequentes que compõe o perfil do paciente aderente são:

1. Idade mais avançada.

2. Etnia branca.

3. Maior escolaridade.

4. Acesso a meios de comunicação.

5. Não tabagistas.

6. HAS em estágios avançados.

7. Em uso de medicamentos na primeira consulta.

8. Não obesos.

9. Referenciados por clínicos ou outros profissionais.

\section{FATORES DE ADESÃO RELACIONADOS AO MEDICAMENTO}

Muitas ações foram adotas ao logo do tempo para elevar a taxa de adesão ao tratamento medicamentoso, entre elas, esforços para minimizar os efeitos adversos das drogas, propostas para simplificação dos regimes terapêuticos, adoção de intervenções para melhorar a motivação dos pacientes e abordagens com foco na educação do paciente. Todos esses itens foram demonstrados na revisão do Cochrane Collaboration, chegando à conclusão de que as intervenções mais eficazes são combinações de estratégias individuais. ${ }^{16}$

Muitos estudos clínicos demonstraram que a média da aderência aos medicamentos anti-hipertensivos era significativamente maior nos pacientes que os tomavam em dose única diária, em comparação com os que tomavam mais do que uma dose por dia. ${ }^{17}$ Este é o aspecto de maior relevância na adesão do paciente ao tratamento medicamentoso, de forma que a adesão reduz drasticamente quando a administração é realizada em duas, três ou mais doses ao dia (Figura 2). Relatos de estudos observacionais revelam que para regimes terapêuticos com uma única tomada diária a taxa de adesão

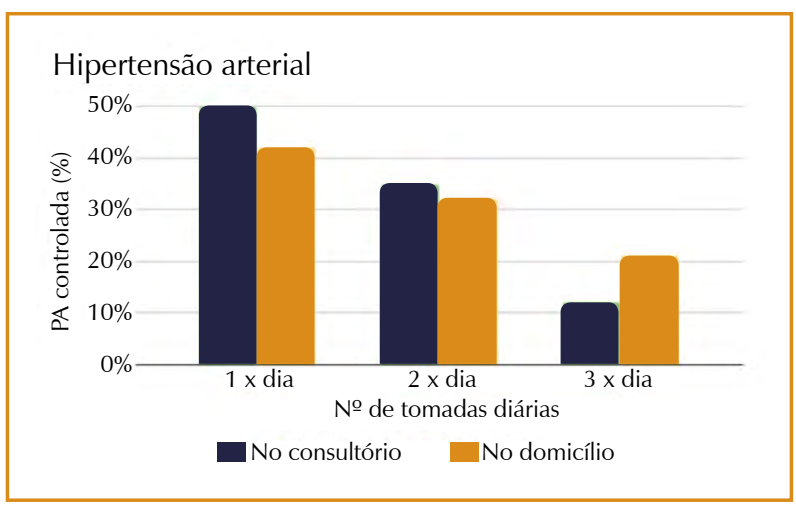

Figura 2. Redução no controle da pressão arterial (PA) com aumento no número de tomadas (Estudo SHEP).

fica em torno de $79 \%$ e que ocorre uma redução progressiva para $69 \%$, 65\% e $51 \%$ nos regimes com duas, três ou até quatro doses diárias respectivamente. ${ }^{18}$

\section{ESTRATÉGIAS PARA ELEVAR A ADESÃO \\ Adesão ao tratamento farmacológico}

Muitas das estratégias testadas em pacientes hipertensos foram compiladas numa meta-análise que resultou numa publicação com 38 estudos que avaliaram 58 intervenções diferentes em mais de 15.000 pacientes. ${ }^{18}$ As evidências apresentadas nesta publicação indicaram as principais medidas que impactam no aumento da adesão ao tratamento medicamentoso, tendo como destaques:

Simplificar o tratamento, evitando a utilização de fármacos que necessitam de várias tomadas ao dia.

Utilizar medidas educativas, informando o caráter incurável da hipertensão primária e os resultados esperados com o tratamento.

Observar as circunstâncias sociais, acesso ao sistema de saúde e condição para aquisição dos medicamentos.

Optar por prescrição de fármacos com menor probabilidade de efeitos indesejáveis

\section{Adesão ao tratamento não farmacológico}

A adesão às medidas do tratamento não farmacológico depende em grande parte da disposição do indivíduo em concordar com mudança em hábitos e condições pessoais que carregam consigo há muitos anos e por isso mesmo são parâmetros onde existe uma grande resistência às interferências. Obter adesão neste campo trata-se de uma missão que requer uma dedicação persistente do médico, sendo recomendável que as metas planejadas sejam negociadas com cada paciente, pois modificações pessoais indicadas de forma consensual possibilitam uma maior chance de resultado positivo na adesão.

Mesmo sendo uma atuação que demanda tempo e energia, o médico não pode se omitir desta atividade pelo importante ganho que existe na redução tensional e na morbimortalidade cardiovascular. O médico tem a obrigatoriedade de abordar durante as consultas aspectos relacionados à adequação do estilo de vida, hábitos e alimentação inadequados, pois existem publicações que revelam que muitas vezes esses assuntos não são ventilados nos atendimentos médicos (Figura 3). ${ }^{20}$ 
Distribuição quanto ao recebimento de orientação para a prática de exercício físico

Sim

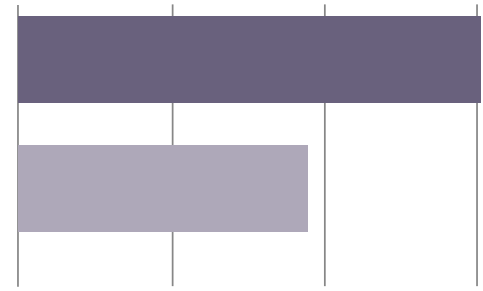

$0,00 \%$

$20,00 \%$

$40,00 \%$

$60,00 \%$

Figura 3. Percentual de pacientes que receberam orientação para atividades físicas (EFDeportes).

Se o tempo reservado para cada consulta médica é muito curto ou as estratégias aplicadas isoladamente não alcançam o resultado esperado para a adesão às medidas não farmacológicas, existe a alternativa de lançar mão das atividades de uma equipe multidisciplinar, contemplando se possível, a participação de enfermeira, psicóloga, nutricionista, assistente social e farmacêutico. Uma equipe assim garantirá um maior sucesso das estratégias adotadas, pois permite ações de intervenção na pós-consulta, avaliação de barreiras socioeconômicas, orientação do uso correto dos medicamentos, adequado acondicionamento dos fármacos e informações sobre higiene alimentar, medidas que no conjunto podem elevar em até $18,5 \%$ a adesão ao tratamento. ${ }^{21}$

\section{Outras estratégias para elevar a adesão}

O rápido desenvolvimento das tecnologias da informação e comunicação fez crescer nesses últimos tempos seu emprego nos serviços de saúde com o objetivo de melhorar os resultados clínicos. A utilização dessas estratégias, indiscutivelmente, permite o aumento na taxa de adesão ao tratamento da HAS com inúmeros potenciais. Dentre as possibilidades, a mensuração eletrônica de doses é um importante recurso desta tecnologia. ${ }^{22} \mathrm{~A}$ adoção desta técnica permite uma monitorização da adesão que pode levar a uma redução ou mesmo a normalização dos níveis de PA em pacientes hipertensos resistentes ao tratamento, ${ }^{23}$ como comprovam resultados de estudos e ensaios clínicos recentes. ${ }^{24}$ Alguns estudos clínicos lançam mão de embalagens inteligentes, empregadas no controle da adesão para medicamentos especiais. A tecnologia utilizada é um sistema conectado ao frasco de medicamentos onde um sensor detecta quando o frasco foi aberto, inferindo-se que a medicação foi tomada e os dados sobre frequência de uso são compartilhados com o médico assistente. ${ }^{25}$

Outro aspecto favorável é a utilização das tecnologias para facilitar a comunicação entre os profissionais e os pacientes, com serviços de endereço eletrônico, reposição da medicação, apoio farmacêutico clínico e ainda cursos interativos. ${ }^{26}$ Os programas com adoção de controle utilizando o emprego de mensagens por telefone, e-mail ou outras vias da internet mostram viabilidade e eficácia, notadamente aqueles instituídos para o controle remoto de pessoas com sobrepeso e obesidade. Muitos resultados positivos foram demonstrados numa série de ensaios randomizados, havendo manutenção por longo prazo da perda de peso e da motivação dos participantes desses programas. Essas intervenções auxiliaram uma perda de peso de 4-7 kg em seis meses a um ano, para o grupo que recebia suporte via e-mail, mensagens automatizadas ou salas de bate-papo em comparação com o grupo onde o controle era realizado apenas nas consultas de revisão. ${ }^{27}$

\section{CONCLUSÃO}

A má adesão à terapêutica anti-hipertensiva constitui um importante obstáculo para obter o controle tensional e alcançar as metas pressóricas estabelecidas nas diretrizes, acarretando insucesso na redução da morbimortalidade cardiovascular. Necessário se faz o emprego constante de estratégias para elevar a taxa de adesão ao tratamento, desde o primeiro contato com o paciente, e manter ao longo do acompanhamento um nível de atenção para a motivação destes, principalmente naqueles com perfil característico para baixa adesão. É mandatório a todos profissionais de saúde procurar identificar as características da sua população assistida, observando todas as variáveis que resultam em não adesão às orientações terapêuticas, procurando reconhecer a real condição socioeconômica das pessoas, buscando individualizar as recomendações de acordo com a perspectiva de cada indivíduo, não perdendo de vista a possibilidade de adotar um acompanhamento realizado por equipe multidisciplinar.

\section{REFERÊNCIAS}

1. Scala LCN, Magalhães LBNC, Machado CA. Epidemiologia e prevenção primária da hipertensão arterial. In: Paola AAV, Barbosa MB, Guimarães JI. Cardiologia. Livro Texto da Sociedade Brasileira de Cardiologia. Barueri: Manole; 2012. p. 235-8.

2. Kearney PM, Whelton M, Reynolds K, Muntner P, Whelton PK, He J. Glopbal burden of hypertension analysis of worldwide data. Lancet. 2005;365:217-23.

3. Turnbull F. Effects of different bloodpressure-lowering regimens on major cardiovascular events: results of prospectively-designed overviews of randomised trials. Lancet. 2003;362(9395):1527-35

4. Chobanian AV, Bakris GL, Black HR, ., et al. The Seventh Report of the Joint National Committee on Prevention, Detection, Evaluation, and Treatment of High Blood Pressure: the JNC 7 report. JAMA. 2003;289(19):2560-72.

5. Hajjar I, Kotchen TA. Trends in prevalence, awareness, treatment, and control of hypertension in the United States, 1988- 2000. JAMA. 2003;290(2):199-206.

6. Turnbull F. Effects of different bloodpressure-lowering regimens on major cardiovascular events: results of prospectively-designed overviews of randomised trials. Lancet. 2003;362(9395):1527-35.

7. Mohan S, Campbell NR. Hypertension management in Canada: good news, but important challenges remain. CMAJ. 2008 ;178(11):1458-60.

8. Sokol MC, McGuigan KA, Verbrugge RR, Epstein RS. Impact of medication adherence on hospitalization risk and healthcare cost. Med Care. 2005;43(6):521-30.

9. Car MR, Pierin AMG, Aquino VLA. Estudos sobre a influência do processo educativo no controle da hipertensão arterial. Rev Esc Enf USP. 1991;25:259-69.

10. Haynes RB. A critical review of the "determinants" of patient compliance with therapeutic regimens. In: Sackett DL, Haynes RB (eds). Compliance with therapeutic regimens. Baltimore, Johns Hopkins University Press, 1976, pp 27-39.

11. Giorgi DMA. Estudo sobre algumas variáveis que influenciam a aderência ao tratamento em hipertensão arterial. Tese de Doutorado. Faculdade de Medicina da Universidade de São Paulo, 1989. 
12. Patel RP, Taylor SD. Factors affecting medication adherence in hypertensive patients. Ann Pharmacother. 2002;36:40-5

13. Heurtin-Roberts $S$, Reisin E. The relation of culturaçy influenced lay models of hypertension to adherence with treatment. Am J Hypertens. 1992;5:787-92.

14. Haynes RB. Determinants of compliance: The disease and the mechanics of treatment. Baltimore MD, Johns Hopkins University Press, 1979.

15. Cramer J. Identifying and improving compliance patterns. In: Cramer JA, Spilker B. Patient compliance in medical practice and clinical trials. New York: Raven Press. 1991:387-392.

16. Haynes RB, Yao X, Degani A, Kripalani S, Garg A, McDonald HP. Interventions to enhance medication adherence. Cochrane Database Syst Rev. 2005(4):CD000011

17. Iskedjian M, Einarson TR, MacKeigan LD, . et al. Relationship between daily dose frequency and adherence to antihypertensive pharmacotherapy: evidence from a metaanalysis. Clin Ther. 2002;24(2):302-16.

18. Claxton AJ, Cramer J, Pierce C. A systematic review of the associations between dose regimens and medication compliance. Clin Ther. 2001; 23(8):1296-1310.

19. Schroeder K, Fahey T, Ebrahim S. How can we improve adherence to blood pressure-lowering medication in ambulatory care? Arch Intern Med. 2004;164:722-32
20. Coelho JESF, Faria FR, Pacheco LF Conhecimento do exercício físico como recurso terapêutico por indivíduos diabéticos tipo 2. EFDeportes. com, Revista Digital. Buenos Aires: 2010:15-150.

21. Logan AG, Achber C, Milne B, Campbell WP, Haynes RB. Work-site treatment of hypertension by specially trained nurses. Lancet. 1979;2:1175-8.

22. Wetzels GE, Nelemans PJ, Schouten JS, et al. Electronic monitoring of adherence as a tool to improve blood pressure control. A randomized controlled trial. Am J Hypertens. 2007;20(2):119-25

23. Green BB, Ralston JD, Fishman PA, et al. Electronic communications and home blood pressure monitoring (e-BP) study: design, delivery, and evaluation framework. Contemp Clin Trials. 2008;29(3):376-95.

24. Adamson SC, Bachman JW. Pilot study of providing online care in a primary care setting. Mayo Clin Proc. 2010;85(8):704-10.

25. Paes AHP, Baker A, Soe-Agnie CJ. Measurement of patient compliance. Pharm World Sci. 1988;20(2):73-7.

26. Blanchet KD. Innovative programs in telemedicine: Great Plains Telehealth Resource and Assistance Center. Telemed J E Health. 2008;14(9):870-4.

27. Tate DF. A series of studies examining Internet treatment of obesity to inform Internet interventions for substance use and misuse. Subst Use Misuse. 2011;46(1):57-65. 